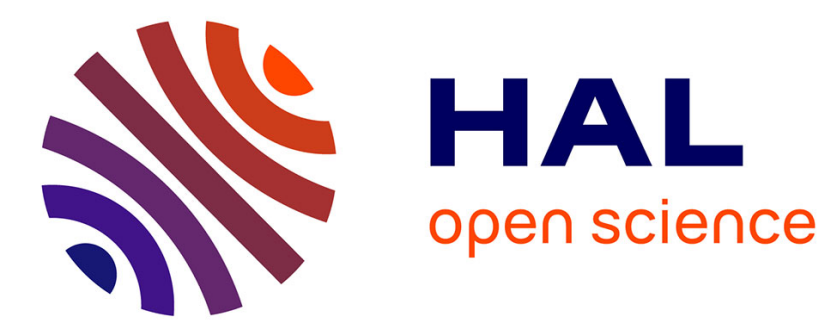

\title{
Embodied gesture interaction for immersive maps
}

Rhys Newbury, Kadek Ananta Satriadi, Jesse Bolton, Jiazhou Liu, Maxime

Cordeil, Arnaud Prouzeau, Bernhard Jenny

\section{To cite this version:}

Rhys Newbury, Kadek Ananta Satriadi, Jesse Bolton, Jiazhou Liu, Maxime Cordeil, et al.. Embodied gesture interaction for immersive maps. Cartography and Geographic Information Science, 2021, 48 (5), pp.417-431. 10.1080/15230406.2021.1929492 . hal-03482497

\section{HAL Id: hal-03482497 \\ https://hal.science/hal-03482497}

Submitted on 16 Dec 2021

HAL is a multi-disciplinary open access archive for the deposit and dissemination of scientific research documents, whether they are published or not. The documents may come from teaching and research institutions in France or abroad, or from public or private research centers.
L'archive ouverte pluridisciplinaire HAL, est destinée au dépôt et à la diffusion de documents scientifiques de niveau recherche, publiés ou non, émanant des établissements d'enseignement et de recherche français ou étrangers, des laboratoires publics ou privés. 


\section{Embodied Gesture Interaction for Immersive Maps}

Rhys Newbury ${ }^{a}$, Kadek Ananta Satriadi ${ }^{a}$, Jesse Bolton ${ }^{\mathrm{a}}$, Jiazhou Liu ${ }^{\mathrm{a}}$, Maxime Cordeil ${ }^{\mathrm{a}}$, Arnaud

Prouzeau $^{\mathrm{ab}}$, and Bernhard Jenny ${ }^{\mathrm{al}}$

${ }^{a}$ Faculty of Information Technology, Monash University, Melbourne, Australia

${ }^{b}$ Inria \& LaBRI (University of Bordeaux, CNRS, Bordeaux-INP)

With the increasing availability of head-mounted displays for virtual reality and augmented reality, we can create immersive maps in which the user is closer to the data. Embodiment is a key concept, allowing the user to act upon virtual objects in an immersive environment. Our work explores the use of embodied interaction for immersive maps. We propose four design considerations for embodied maps and embodied gesture interaction with immersive maps: object presence, consistent physics, human body skills, and direct manipulation. We present an example of an immersive flow map with a series of novel embodied gesture interactions, which adhere to the proposed design considerations. The embodied interactions allow users to directly manipulate immersive flow maps and explore origin-destination flow data in novel ways. Authors of immersive maps can use the four proposed design considerations for creating embodied gesture interactions. The discussed example interactions apply to diverse types of immersive maps and will hopefully incite others to invent more embodied interactions for immersive maps.

Keywords: embodied interaction, embodiment, virtual reality, augmented reality, immersive map, immersive analytics, origin-destination flow map

\section{Introduction}

The recent advancement of graphics hardware technology has led to affordable head- mounted virtual reality (VR) and augmented reality (AR) displays for immersive visualization. VR can create an immersive experience where virtual objects are perceived as being present, and AR can plausibly blend virtual objects with the physical environment. Researchers have explored

\footnotetext{
${ }^{1}$ corresponding author: bernie.jenny@ monash.edu
} 
the benefits of such immersive spaces for information visualization and analysis (Marriott et al., 2018), including immersive maps and other types of geospatial visualizations (for historical overviews, see Hedley, 2015, 2017). The combination of head-mounted displays and interaction technologies - away from the traditional desktop - is an exciting new field for the visual analysis of geographic information. Inspired by these developments, we explore embodied interaction with hand gestures for immersive maps.

Immersive analytics is an emerging field that explores embodied data visualization and analysis in immersive space (Chandler et al., 2015; Dwyer et al., 2018). Immersive analytics applies "engaging, embodied analysis tools to support data understanding and decision making" (Dwyer et al., 2018) using VR or AR head-mounted displays and input with hand-held controllers or hand gestures.

Embodiment is an important concept for immersive analytics that opens up the opportunity to exploit users' proprioception - the sense of self-movement and body position for data visualization and data analysis (Mine et al., 1997). A series of recent works have proposed embodied information visualization, for example, by using embodiments of abstract data axes in immersive space (Cordeil et al., 2017, 2019; Sicat et al., 2019), three-dimensional trajectories (Hurter et al., 2018), and time-space trajectories (Wagner Filho et al., 2020). A pioneering example is the ImAxes VR framework by Cordeil et al. (2017) that uses immersive analytics for decision making with abstract data. ImAxes embodies axes of abstract data as virtual sticks that can be grabbed and manipulated. The user combines multiple embodied axes to create non-geospatial visualizations, such as three-dimensional scatter plots or parallel coordinate plots.

Previous works in immersive analytics have demonstrated the benefits of embodied interactions for knowledge discovery. For example, Hurter et al. (2018) showed that their immersive visualization system, Fiberclay, can help experts to identify anomalies in flight traffic data. Users of Fiberclay intuitively manipulate three-dimensional trajectories with hand gestures. Butscher et al. (2018) demonstrated how AR tabletop visualization can support collaborative visual analysis for nutritional science experts by utilizing multimodal tabletop and head-mounted displays. Immersive analytics has also been studied in other fields, including, but not limited to, economy (Batch et al., 2020), energy visualization (Ens et al., 2020), and factory safety (Prouzeau et al., 2020). For and exhaustive review see the recent paper by Ens et al. (2021).

Research in immersive maps and geovisualization picked up some of these ideas for embodied visualization with maps (for a recent overview see Çöltekin et al., 2020). Examples include 3D flow maps (Yang et al., 2019; Zhang et al., 2016, 2018), bar graphics in virtual landscapes and on maps (Quach \& Jenny, 2020), choropleth maps that transition to prism maps 
and bar charts (Yang et al., 2020), space-time cubes (Wagner Filho et al., 2020), threedimensional trajectories (Hurter et al., 2018) and streamlines (Bilke et al., 2014), as well as a side-by-side comparison of immersive geovisualizations (Nam et al., 2019). Researchers also explored how to best interact with (Austin et al., 2020; Giannopoulos et al., 2017; Santos-Torres et al., 2018; Satriadi et al., 2019) and arrange (Satriadi et al., 2020; Spur et al., 2020) maps in immersive space.

This paper focuses on interacting with embodied maps and geovisualizations in virtual reality and augmented reality. Our exploration of embodied interaction for maps is inspired by the embodiment of data in ImAxes. We adapt and extend these ideas to immersive maps and geovisualization. This paper makes two contributions. First, we explore the use of embodiment for immersive maps, and we identify four design considerations for embodied maps and embodied interaction. Second, we explore these design considerations to create a series of novel direct embodiment interactions. We achieve this by demonstrating and exploring how embodiment and embodied interactions can be used for immersive flow maps. While our example uses an immersive origin-destination flow map, the embodied interactions are transferable to other types of immersive maps.

Figure 1 illustrates some of our ideas for embodied interaction using an immersive origin-destination flow map example. Pushing multiple flow links (Figure 1 top left) reveals short or thin flows occluded by long and thick ones. Shaking the map (Figure 1 top right) filters out flows representing small values to reduce visual clutter. Pulling out a flow line (Figure 1 bottom left) instantiates a new flow map showing more detailed flows. Moving two maps close together (Figure 1 bottom right) creates flow links connecting the two maps.

Our goal is to offer the user a fluid and effortless flow of embodied interactions (Elmqvist et al., 2011) to create maps with varying levels of detail and perform a series of analysis steps. For example, the user may pull out two flows from a state-level map to create two new county-level maps; then bring these two maps together to connect them with flow lines; shake the maps to remove small flows; and push away flows to reveal an interesting cluster of previously hidden flows. 

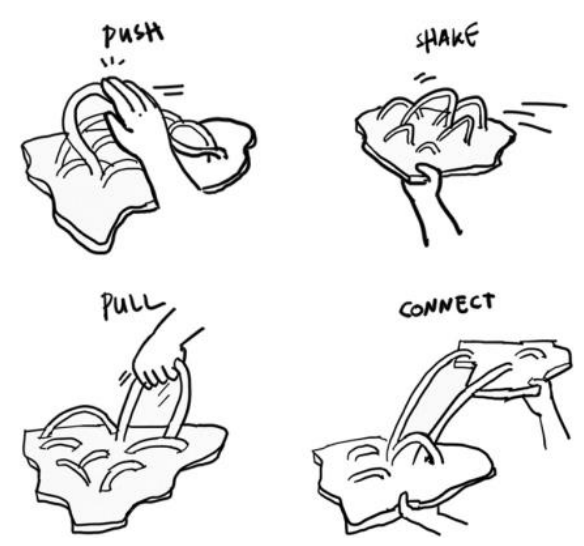

Figure 1. Examples of embodied interactions for immersive flow maps: pushing flow lines to reveal hidden flow lines, shaking the map to filter small flows, pulling out a flow line to create a detail map, and connecting maps with flow lines by bringing them together.

To simplify the discussion in this paper, we use the term "embodied map" to refer to maps, globes, and other types of geovisualizations in virtual reality and augmented reality that use embodiment. Section 2 reviews embodiment for immersive visualization and related background concepts for immersive analytics. Section 3 identifies design considerations for embodied maps. Section 4 presents the example immersive flow map and discusses the embodied interactions. The discussion in Section 5 identifies the design considerations applied to the immersive example map, before the paper concludes in Section 6.

\section{Background: Immersion, Presence, Embodiment, and Embodied Interaction}

Important concepts in immersive visualization include immersion, presence, embodiment, and embodied interaction. We discuss these interconnected concepts here, because they are not commonly considered in cartography and geovisualization and are sometimes used inconsistently (Klippel, 2020).

Immersion and presence are two concepts related to embodiment but are also clearly distinct from it. The degree of immersion of a visualization is determined by the technology used: the more vivid the illusion of reality to the human senses, the higher the immersion (Slater \& Wilbur, 1997). In immersive visualization, the display system and the use of body tracking (through head-mounted displays and tracked controllers) aim to increase the degree of immersion for the user (Laha et al., 2012). Immersion is a requirement for creating a sensation of presence.

Presence is "the subjective experience of being in one place or environment even when physically situated in another" (Witmer \& Singer, 1998). Immersive technologies can produce spatial presence with true-to-life simulations (Dwyer et al., 2018). These immersive simulations 
aim at creating a strong perception of presence - a feeling of 'being there', which results in virtual objects being experienced as actual objects (K. M. Lee, 2004). Dwyer et al. (2018) describe many factors that can affect the user's feeling of presence in immersive space, including: inclusiveness (the degree to which the virtual world blocks out the real world), extensiveness (a range of sensory channels), vividness (visual realism or fidelity), and plausibility (the extent to which the objects and actors exhibit real world behavior). For example, ImAxes creates a sense of presence using inclusiveness and vividness; ImAxes does not aim to plausibly recreate the world in a true-to-life simulation. Our work aims to create a similar sense of presence for immersive maps and geovisualizations by adapting these factors.

Embodiment can be seen as "the sense of self-location, the sense of agency, the sense of body ownership" (Kilteni et al., 2012). If the user sees through the eyes of a virtual body and this virtual body acts in concert with the user, then the virtual body is perceived as being spatially coincident with the user's physical body and "embodies" the user in the virtual world (Falconer et al., 2014; Kilteni et al., 2012). Others have defined embodiment more broadly. Dourish (2001a) includes "things that unfold in the world", which are mainly virtual objects that represent an entity and can be acted upon (e.g. picked up, examined, manipulated, or rearranged). We adopt Dourish's definition for our exploration of embodied interaction for immersive maps.

The concept of embodied interaction (Dourish, 2001) is the answer to the fact that traditional desktop interfaces with a mouse and keyboard do not transfer well to immersive spaces, because they are not designed for object manipulation in three-dimensional space. Embodied interaction can use dedicated tangible controllers, such as physical sliders (Cordeil et al., 2020; Walsh et al., 2018), but more commonly uses virtual and highly abstract objects, such as the virtual data axes of ImAxes (Cordeil et al., 2017). In immersive space, embodied interaction is a direct manipulation style for rapid interaction (Shneiderman et al., 2016, p. 214).

Direct manipulation of objects is also a natural interface, that is, an interface that “makes users act and feel natural" (Wigdor \& Wixon, 2011, p. 14). Fishkin et al. (1998) argue that the more the embodied interaction is analogous to a real-world task, the more natural and transparent the interaction becomes. Users carry out embodied interaction by directly manipulating objects with their bodies or performing indirect gestures (Mine et al., 1997). Examples are gestures with hand-held controllers, freehand gestures (e.g. Satriadi et al., 2019), foot gestures (Austin et al., 2020), gestures with embodied tools (e.g. a virtual laser pointer), or a virtual avatar body (Jerald, 2015).

The challenge with immersive maps is to design direct and natural embodied interactions. Büschel et al. (2018) describe different forms of interactions for immersive visualizations: selection, filter, sort, navigation, reconfiguration, and labelling and annotating. In 
immersive space, many of these operations are possible with embodied interaction and have been demonstrated in previous studies. However, embodied interactions for map-specific operations have not been thoroughly explored.

\section{Considerations for Designing Embodied Maps}

We explore four main design considerations for embodied maps in an immersive environment. (1) Object presence: the map should be perceived as an object that is present; (2) consistent physics: the map should follow a set of physical laws; (3) human body skills: the user's basic physical skills should be transferrable; and (4) direct manipulation: the map should support direct manipulation for map-specific operations. We identified these design considerations through a literature review focusing on embodiment applied to non-geospatial data visualization for immersive analytics, building exploratory embodied maps, and practical experimentation with embodied maps in VR and AR. The four design considerations discussed below are propositions that we expect to evolve and develop further, and grow in number, as more advanced technology for immersive visualization and gesture tracking becomes available.

Object presence: An embodied map should be perceived as a three-dimensional

object that is present. This design consideration encourages users to treat virtual objects as physically present such that they interact with the objects. The sense of presence can be achieved by making the affordances of the object more visible (Norman, 2013, chapter 4), i.e., by applying depth cues, such as shading and light reflection, casting shadows, varying size with distance, or realistic occlusion among virtual and physical objects (Ware, 2012). In addition, an embodied map should be modeled as a three-dimensional object. This can be achieved, for example, by giving flat maps a perceptible thickness, or by using virtual globes (Yang et al., 2018).

Consistent physics: An embodied map should follow a set of physical laws. The immersive world in which the map exists should apply a consistent set of laws of physics that affect its virtual objects (Jacob et al., 2008). An embodied map can imitate naïve laws of physics, such as the effect of gravity or the absorption of light. For example, a virtual map in AR may fall onto a physical desk when released, and it may cast shadows on other physical and virtual objects. However, adherence to the laws of physics in immersive space is not a requirement; a virtual map may be subjected to "alternative" laws of physics that do not exist in the real world. For example, embodied maps can be pinned in open space without being affected by the laws of gravity, and maps (or elements of a map) can shrink or grow, change their shape, or vanish. Nevertheless, the set of physical laws applied to the embodied maps should be consistent to provide a clear mental model of the embodied map's behaviors. For instance, 
knowing that maps are floating in the air, users will not hesitate to grab a map and release it at any point in immersive space.

\section{Human body skills: The user's basic physical skills should apply to embodied}

maps. Users should be able to apply basic physical skills to interact with an immersive system (Jacob et al., 2008). This includes basic actions such as grabbing and throwing maps and other virtual objects with their hands or controllers. This enables users to directly act upon a virtual map by picking it up, enlarging it by pulling its corners, or positioning it in space. Walking is another basic physical skill that can be used for immersive visualization. Provided there is an adequate tracking space, users can walk towards or around a virtual map to inspect it at varying distances and from different perspectives (Bruder et al., 2009).

Direct manipulation: An embodied map should support direct manipulation. The fluid interaction concept in visualization theory posits that an interactive system should promote a flow of actions, support direct manipulation, and minimize the effort required to perform actions (Elmqvist et al., 2011). This concept also applies to interaction with embodied maps, and the ability to directly manipulate immersive maps is also suggested by user preferences (Austin et al., 2020). A small set of direct manipulations has been proposed for immersive maps. For example, a rotation gesture can be used to transition between a choropleth map, a prism map, and a bar chart (Yang et al., 2020), a pointing gesture can adjust the projection center of a world map (Yang et al., 2018), or hand gestures can zoom and pan an immersive map (Satriadi et al., 2019; Wagner Filho et al., 2020). These embodied interaction examples affect the entire map or individual components of an immersive map. The individual components - such as a map symbol, a map layer, or the legend - can also afford embodied interactivity with direct manipulation. For example, with direct manipulation, an individual map symbol can display information when tapped (Wagner Filho et al., 2020), a point marker can be added with a pointing gesture (Austin et al., 2020), or the base plane of a space-time cube can be adjusted (Wagner Filho et al., 2020).

\section{An Immersive Flow Map with Embodied Interaction}

\subsection{Design Approach}

We applied the design considerations described in Section 3 to create an interactive flow map visualization in immersive space to invent new embodied interactions for immersive maps that aim to feel "natural" and directly manipulate objects. We focused on exploring innovative embodiment and embodied interactions.

We chose to create an immersive flow map, because the visualization, analysis and interpretation of origin-destination flow datasets is often very difficult due to significant visual 
clutter caused by overlapping flows (Schöttler et al., 2021). Our immersive flow map is inspired by previous work on the three-dimensional visualization of origin-destination flows in virtual reality by Yang et al. (2019), who visualized flows with three-dimensional curved tubes on flat maps and globes in virtual reality. Their work suggested that careful use of the third spatial dimension can reduce visual clutter in complex immersive flow maps. We enhanced their interactivity with additional embodied, body-based interaction.

When ideating embodied interactions, we were initially inspired by embodied interactions in ImAxes as well as the gestures for manipulating immersive maps identified by Austin et al. (2020). We applied an exploratory and iterative methodology: We brainstormed potential interactions, sketched them graphically, then coded them in exploratory prototypes and evaluated the interactions informally. This procedure led to improvements of earlier ideas and generated new ideas for additional interactions. The embodied interactions presented here are those that (1) we found to be natural and relevant, and (2) act on embodied objects that the users perceive as three-dimensional objects.

\subsection{Implementation}

We implemented our design using the Unity game engine (unity.com) with the VRTK Virtual Reality Toolkit (vrtoolkit.readme.io) for interactions and used various state-of-the-art virtual reality headsets.

Our immersive flow map (Figure 2) visualizes U.S. county-to-county migration data from the U.S. Census Bureau (U.S. Census Bureau, 2020). We followed design principles for designing flow maps in 2D (Jenny et al., 2018) and 3D (Yang et al., 2019) to reduce intersecting flow lines and arrange flows in the third dimension. We also took inspiration from the interactive 2D flow map of U.S. migration by Stephen and Jenny (2017). Our immersive map (Figure 2) shows curved flow lines between counties and states using a modification of a constraint-based layout model developed by Prouzeau et al. (2019). They designed their method to minimize visual clutter and occlusion by optimizing the routing of the three-dimensional flow lines. However, we could not directly use the original method because: (1) the flow lines were too slow to react to movements of the map in real-time, and (2) flows appeared flat against the map as there was no force to direct the lines away from the map. Therefore, we increased the amount of physics calculation per frame by extending the original graphics processing unit (GPU) based computation to achieve interactive frame rates. We also added new forces in the normal direction of the map to make the lines appear to curve away from the map. A constant amount of force was applied to each point, and since the number of points increased with the length of the lines, this had the additional effect of making the height of the lines change based on distance: longer lines are higher and shorter lines are lower, which improves readability as 
suggested by Yang et al. (2019). Finally, we increased the repulsing forces between the lines to encourage more separation between lines.

The flow map varies the thickness of flows with their magnitude as in the immersive flow maps by Yang et al. (2019). We indicate the flow origin with green and the flow destination with blue. The map additionally shows population density with a choropleth map, uses the Albers equal-area projection, and its base extrudes into the third dimension to create an impression of a three-dimensional object.

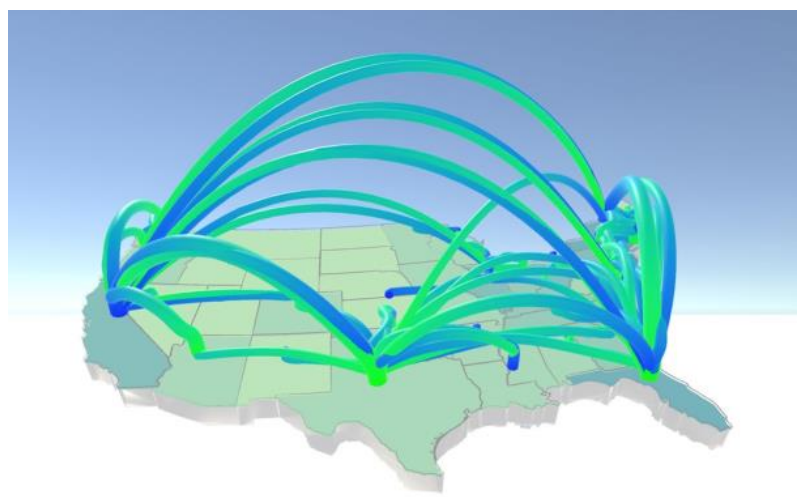

Figure 2. $\quad$ Flow map showing the largest 100 migration flows between U.S. states in virtual reality. The color gradient of the flows indicates flow direction (from green origins to blue destinations).

\subsection{Embodied Interactions}

We designed our interactions for freehand gestures, which is the most natural way to interact with real or virtual objects. However, we implemented all interactions for standard hand-held VR controllers and left the detection and interpretation of freehand gestures for future work.

We map the embodied gesture interactions to enabling operators and work operators (Roth, 2013). Enabling operators prepare for the use of work operators or clean up after using work operators, while work operators are productive activities that help accomplish the desired objective (Whitefield et al., 1993; Roth, 2013). Table 1 lists the embodied gestures, their type (i.e., enabling or work operator) and interaction operator. For example, the first row documents a gesture that grabs and moves a map, which is translated to an enabling operator that positions and orients the map in space. The enabling operators include operators for positioning maps, creating and deleting maps, linking and unlinking maps, and adjusting the size of maps.

Examples of work operators include a filter, a reveal and a retrieve operator. The following paragraphs illustrate the operators in Table 1.

It is to note that some operators can be considered as enabling or work operators, depending on the context in which they are used and the task to solve, as shown by Davies 
(1998) in a discussion of enabling and work operators for GIS tasks. For example, the orient and position operators, which we consider as enabling operators, could be classified as work operators.

Some interaction operators apply to a single map, while other operators require two or more maps. For example, the delete operator uses a throwing gesture that is applied to a single map, while the link operator creates linking connections between multiple maps.

Table 1: Direct embodied gestures for immersive maps and their mapping to enabling operators (first) and work operators (last).

\begin{tabular}{lll}
\hline Embodied Gesture & Operator Type & Interaction Operator \\
\hline Grabbing and moving a map & Enabling & Orient and position \\
Pulling out a map region or a map symbol & Enabling & Create \\
Throwing a map & Enabling & Delete \\
Two-handed grabbing of two maps then & Enabling & Link (connect maps with \\
bringing them together & & links) \\
Two-handed grabbing of two maps then & Enabling & Unlink (remove links \\
moving them apart & & between maps) \\
Two-handed grabbing gesture & Enabling/Work & Zoom (geometric and \\
& & semantic) \\
Grabbing and shaking a map & Work & Filter by quantitative \\
& & attribute
\end{tabular}

Grabbing and moving to orient and position a map: The embodied map supports direct manipulation through direct grabbing. This allows the user to grab and then freely orient and position the map in space (as shown in Figure 3). Once the user releases the map, the map will remain stationary, appearing pinned in open space without being affected by gravity. 


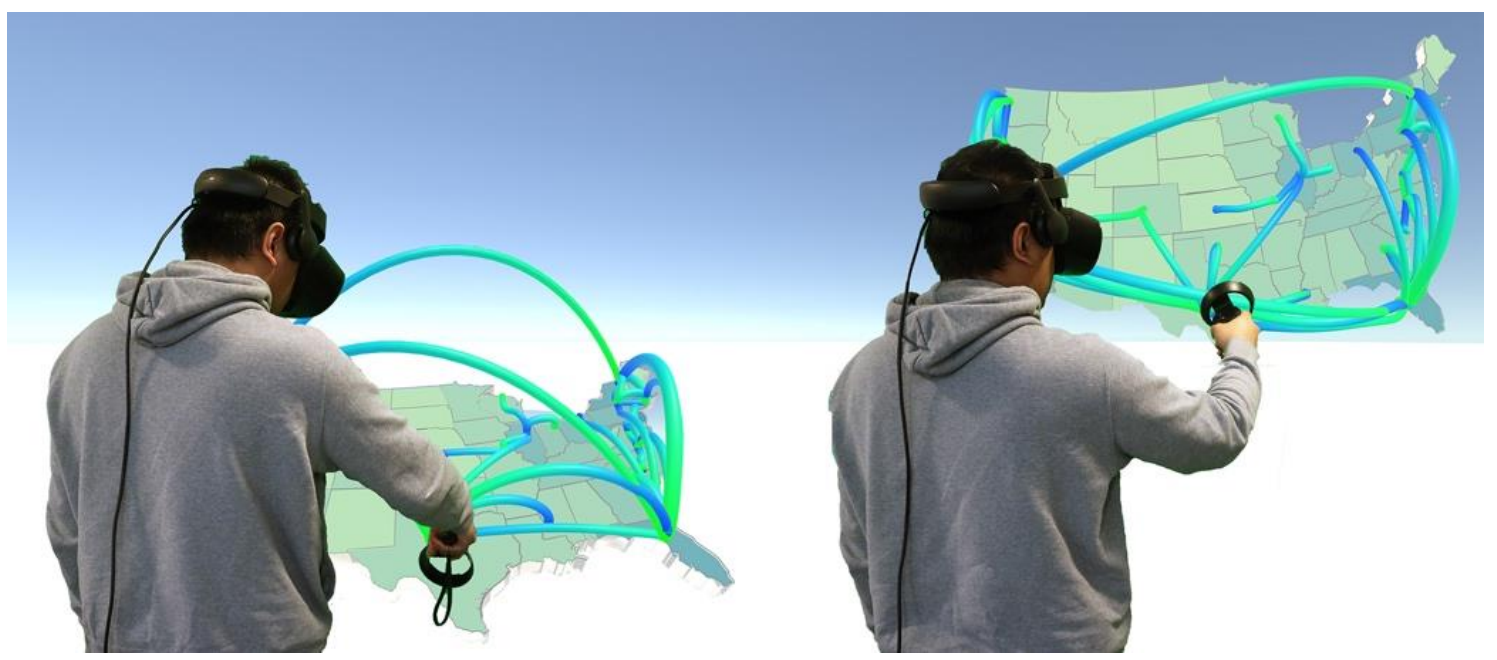

Figure 3. Embodied grabbing and moving gesture for orienting and positioning a map; here the map is lifted and oriented vertically.

Pulling a map region or a map symbol to create a new map: By grabbing and pulling a region or a map symbol, a new map is created. The new map shows the pulled region with more details. Figure 4 shows the user gaining detailed information about the state of Texas by "pulling out" the geometry of that state. The example in Figure 4 creates a new map showing the population density and county-to-county migration in Texas. Alternatively, users can grab a map symbol, such as a flow line, and then "pull it out" of the map to create a new map to more closely inspect the area of the symbol. Once the interaction is completed, the symbol that was pulled out reappears at the original location, such that the original map is not affected. For our flow map, we decided to create two maps when a flow line symbol is pulled; one map for the origin region and one map for the destination region. For example, when the user pulls out a state-level flow line between California and Texas, a map for both states is created and the county-level flow lines between the two maps are shown (Figure 5). Map slicing is an alternative to pulling out a predefined region or symbol; it consists of defining an area typically with a rectangular shape - which is then pulled out (Satriadi et al., 2020). Any of these variations follow Shneiderman's information-seeking mantra (Shneiderman et al., 2016), allowing the user to zoom and gain information about specific parts by creating more detailed maps. 


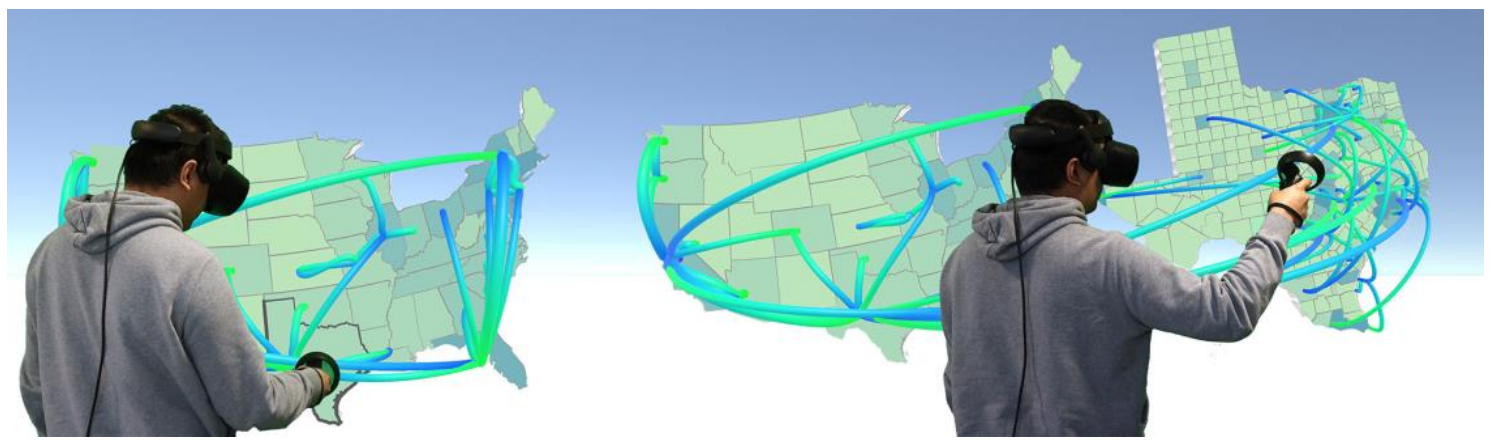

Figure 4. A pulling gesture for creating a detail map. A state is "pulled out" of the map to create a new map of that state with more detailed county-level geometry.

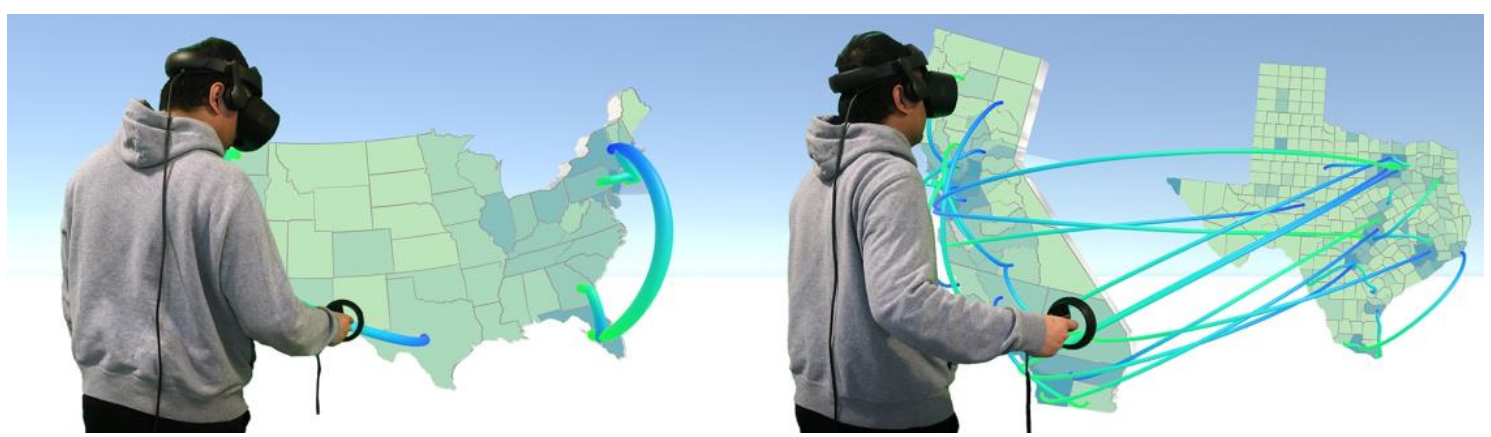

Figure 5. Pulling out flows for creating two detail maps. In this example, the user pulls out the flow between California and Texas, which creates two new maps, one for each end point.

Throwing to delete a map: A map is deleted with a throw-away gesture (Figure 6) that mimics the user throwing away a crumpled piece of paper. Immersive analytics frameworks have used a similar enabling interaction for deleting embodied objects that are no longer needed (Cordeil et al., 2017; Lee et al., 2021). The thrown-away map twirls through the air, sinks into the floor, and disappears. (While Roth (2013) considers the delete interaction to be an edit operator that manipulates the geographic information underlying the map, in our context the delete operator removes the entire map.) 


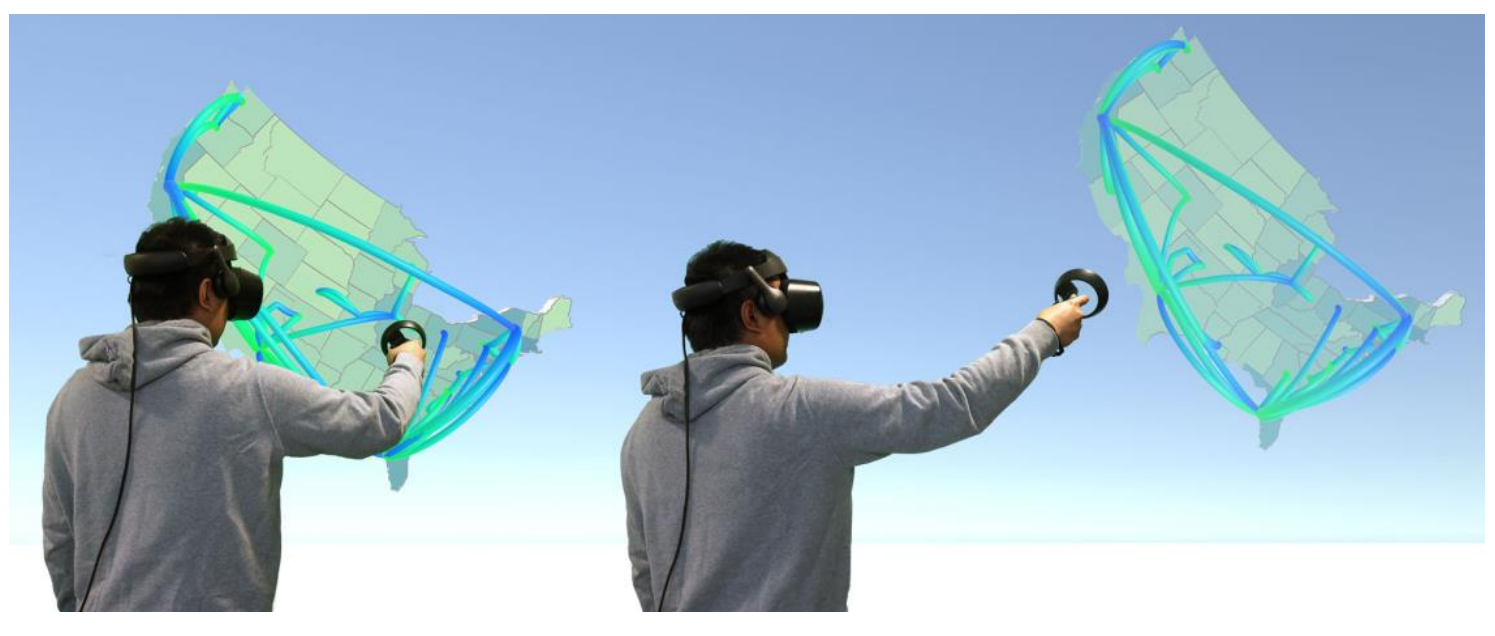

Figure 6. Embodied throw-away gesture to delete a map.

Moving maps together to link maps: When two maps are moved sufficiently close together, links between both maps are displayed (Figure 7, top). This proximity-based interaction is inspired by ImAxes (Cordeil et al., 2017), where parallel coordinate plots are created when two data axes are moved close together. This interaction opens up a series of visualization possibilities, for example, creating chained, webbed, or tree-structured geospatial flow visualizations across multiple maps. Figure 7 (bottom) shows an example where the user has created a chained series of flow maps between different states in the U.S. Connecting maps with visual links is particularly useful for flow maps, because the links can show quantitative flows. However, similar "bringing together" interactions for creating visual links are applicable to other coordinated visualizations. For example, Prouzeau et al. (2019) connect locations on immersive maps with associated statistical diagrams using visual links. 


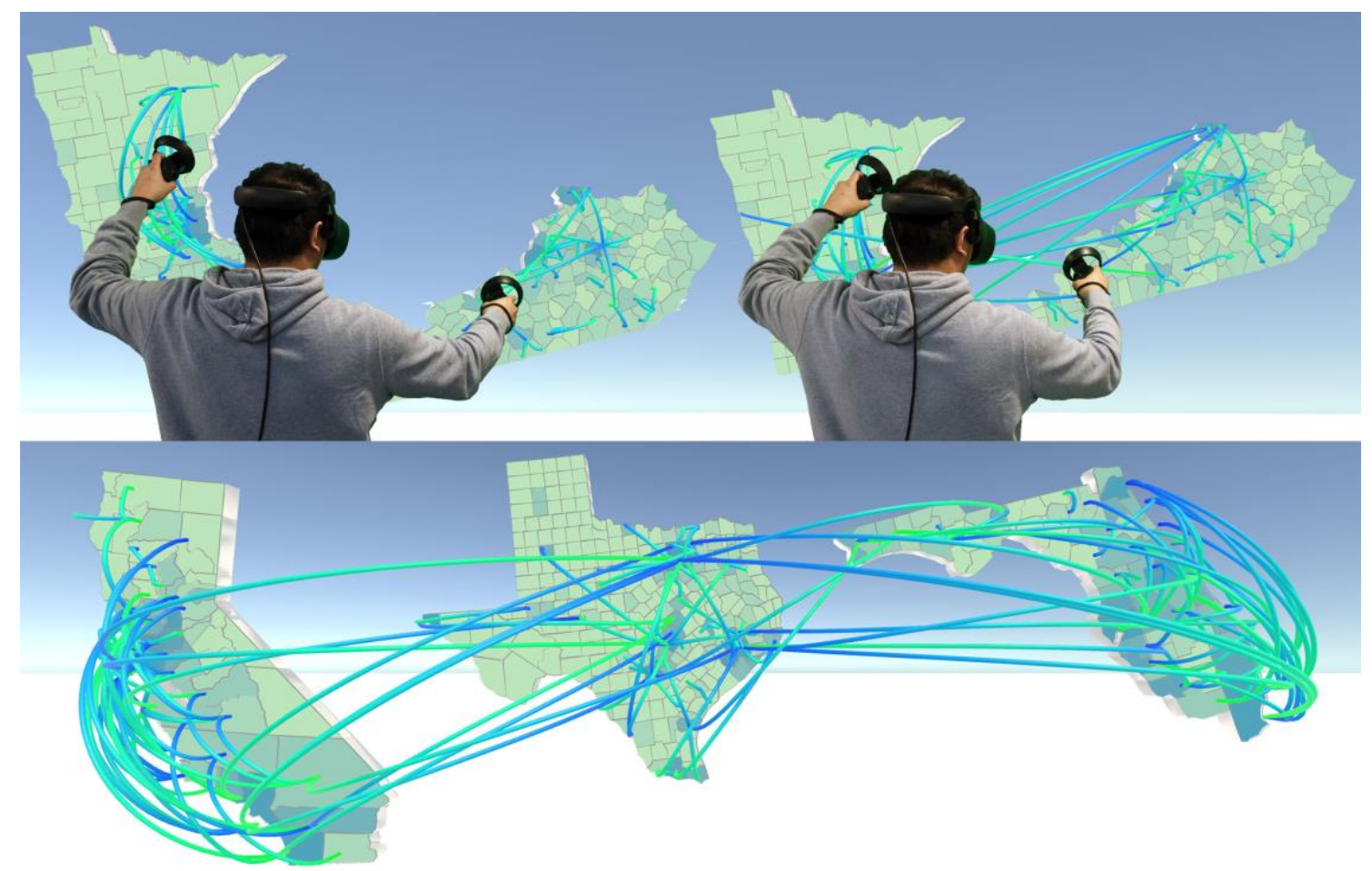

Figure 7. Embodied interaction for creating flow lines between two maps. Top left: maps are moved together. Top right: linking flow lines appear when the two maps are close enough. Bottom: An example of chained flow maps, where the user linked three maps together in a custom arrangement.

Moving a map away from another map to unlink maps: Users can remove the links connecting maps that were created by moving maps together by pulling the two maps apart. This is the opposite of the "moving together" gesture shown in Figure 7 (top). This can be done by pulling both maps apart or by moving one map sufficiently far away from the other.

Two-handed grabbing gesture to zoom a map: The size and scale of an embodied map can be adjusted with a two-handed grabbing gesture on the map followed by moving the hands apart or together (Figure 8). This gesture is similar to the familiar pinch gesture for zooming maps on touch displays and has been the most commonly suggested hand gesture for interacting with immersive maps in an elicitation study by Austin et al. (2020). 


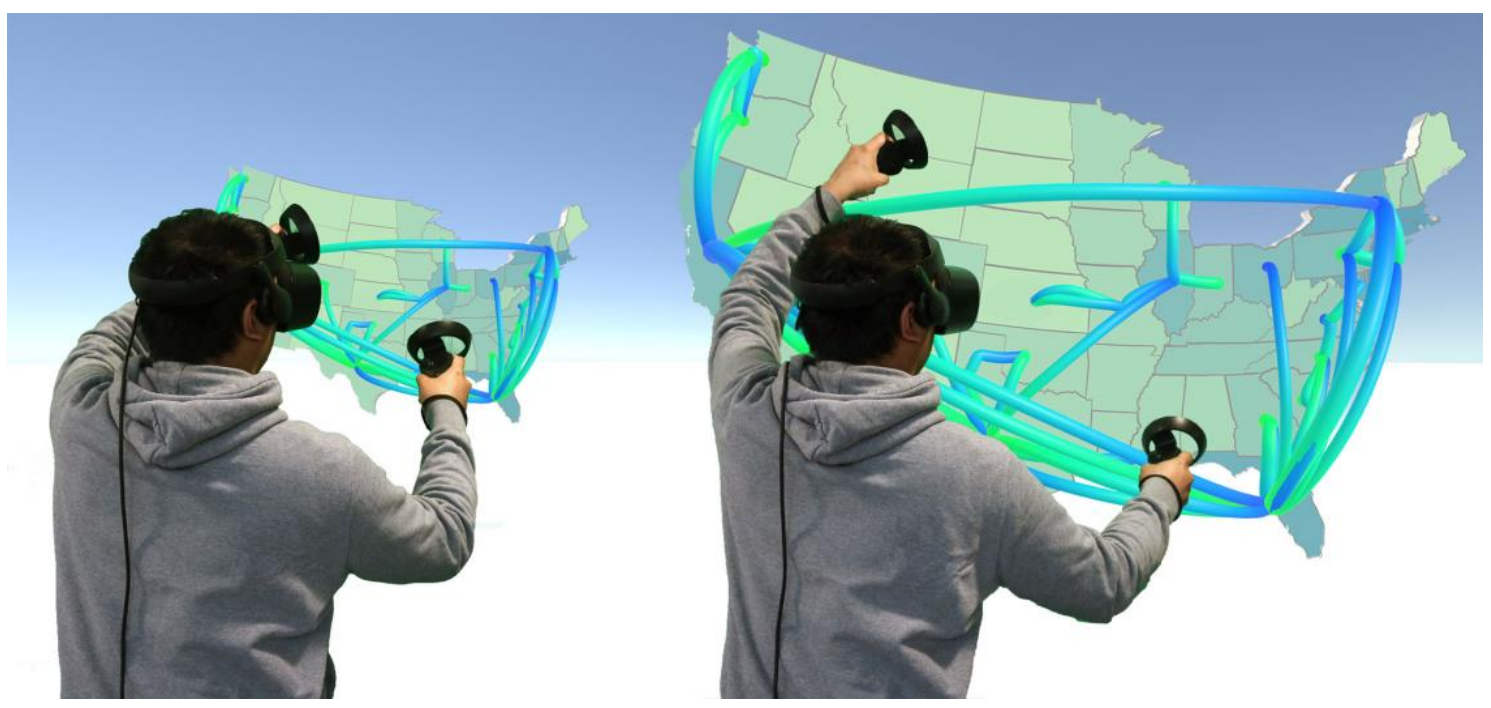

Figure 8. Scaling a map with a bimanual gesture.

Grabbing and shaking a map to filter data: The user can grab the map and perform a shaking gesture with the map. We use this direct manipulation to filter quantitative data represented by proportional map symbols. The longer the map is shaken, the more symbols representing small values are removed. This could affect various types of quantitative symbols, such as length-proportional bars, volume-proportional cubes, or size-proportional flows as in our map (Figure 9). To detect shaking, we find the angle between two consecutive velocity vectors: Angle $=\cos ^{-1}\left(v_{i} \cdot v_{i-1}\right)$. A large angle indicates a change in the direction of the map. We look at a rolling buffer of one second; if there is an angle greater than $30^{\circ}$ within the one second buffer, we consider the map as currently being shaken. If there is no large angle, we consider the shaking to have stopped. Every $0.5 \mathrm{~s}$ of shaking, the $n$ smallest symbols are removed; $n$ is a percentage of the number of initial symbols. This is an ephemeral interaction in our implementation because all symbols return when the user releases the map. The filtering by shaking interaction could be complemented with an animation showing the symbols tumbling out of the map and falling onto the floor to illustrate the number of removed symbols.

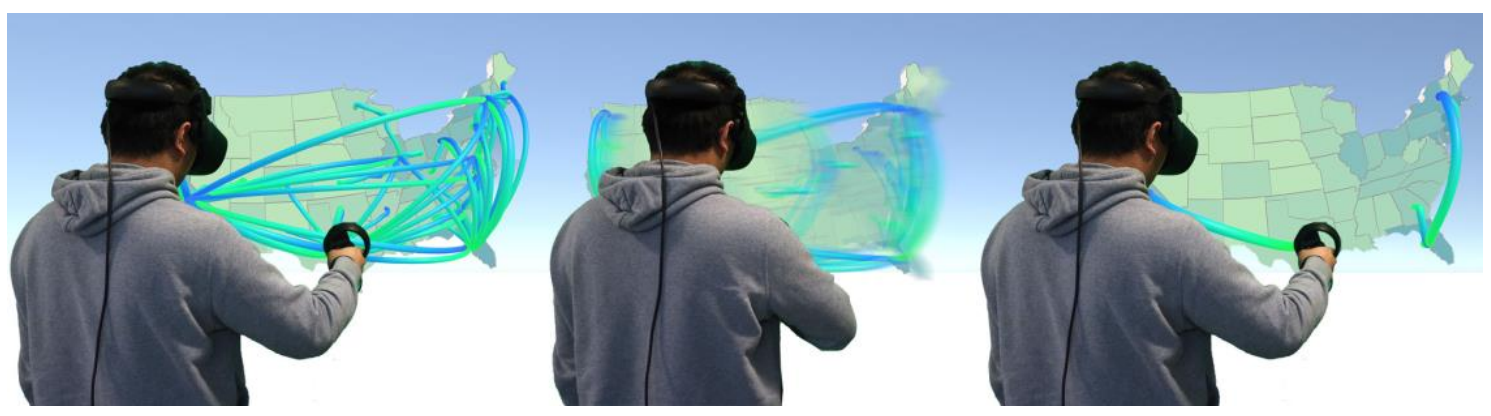

Figure 9. Embodied interaction for filtering by shaking the map. Left: before shaking, the map shows all flow symbols. Center: Shaking removes flows with small values. Right: After shaking, the map only shows flows with large values. 
Pushing away symbols to reveal information: Flow maps and other thematic threedimensional maps can easily become cluttered and suffer from occlusion among symbols. With embodied maps, users can use their hands or controllers to push or bend large symbols that occlude other symbols. For a prism map or a map with three-dimensional bars, large prisms or bars could temporarily be pushed down or bent away to reveal a view of the previously hidden neighboring symbols. Once the gesture is released, the symbols could elastically snap back to their initial position. For our flow map, the pushing force acts in the direction of the velocity of the controller or the tracked hand (Figure 10). For example, if the user moves the hand to the left, the force will only act in this direction, pushing relevant flows to the left. If the controller velocity is smaller than a specified threshold, we latch onto the previous direction, allowing the user to hold the flows after the initial pushing action.

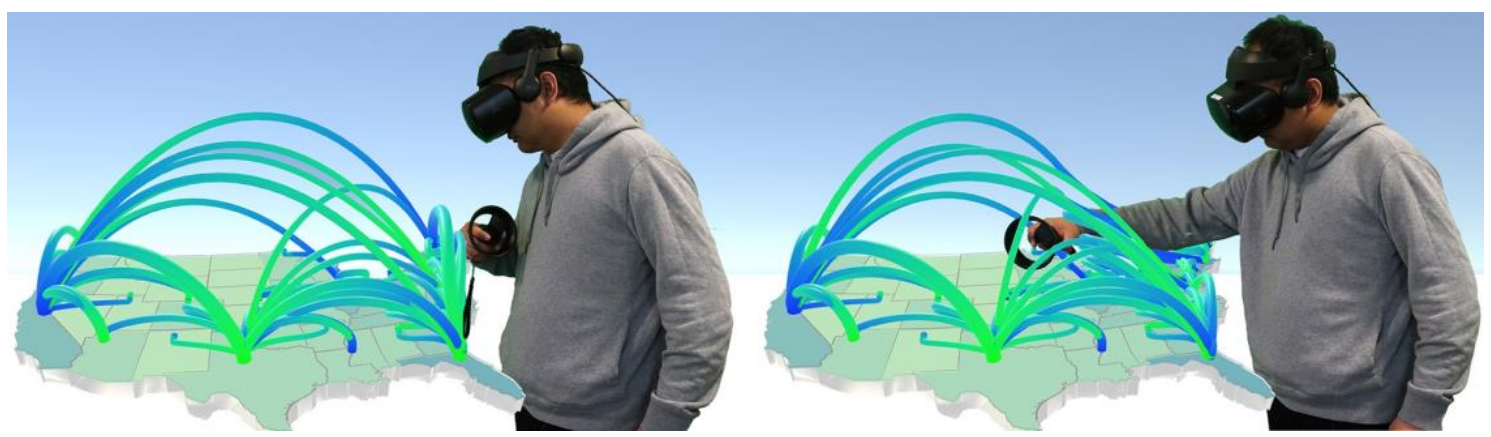

Figure 10. User pushing away a map symbol to reveal hidden information. Left: A thick and long flow starting in Florida and ending in the northeastern U.S. hides shorter and thinner flows. Right: The user is pushing the long flow away with the controller to reveal the smaller flows.

Pointing at or touching a map symbol to retrieve information: Using a laser pointer metaphor, users can point at a map symbol or directly touch a map symbol to retrieve specific information about it. This work operator follows Shneiderman's information-seeking mantra (Shneiderman et al., 2016), allowing the user to gain details on demand about specific parts of the visualization. This is shown in Figure 11, where the details of the flow are displayed close to the controller. 


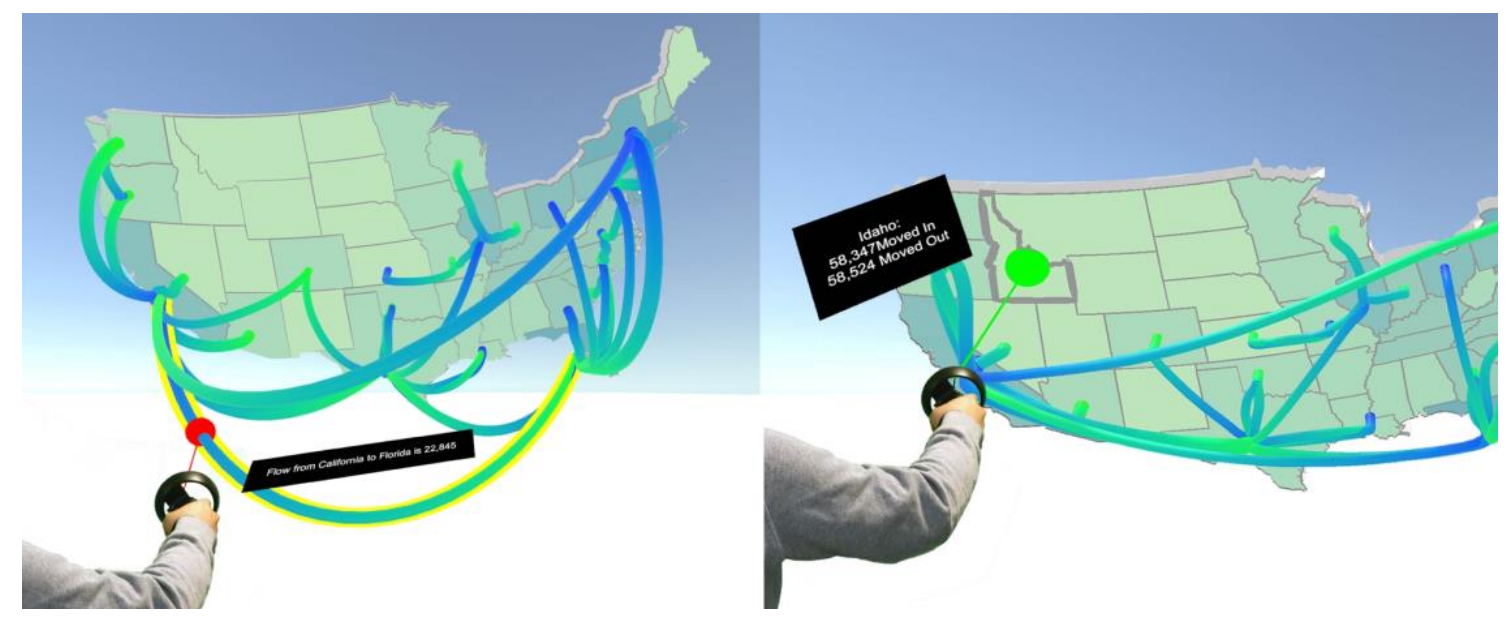

Figure 11. Retrieving information with a laser pointer metaphor for a flow symbol (left) and an area symbol (right).

\section{Discussion}

The first consideration for designing embodied maps in Section 3 is object presence. It suggests showing maps as three-dimensional objects that are perceived as being present. We initially created an infinitely thin base map for our example flow map and showed flows with twodimensional bands. We quickly realized that such a visualization only conveys a weak sense of presence, and we found that the thin choropleth map and the two-dimensional flow bands were not enticing the user to explore the use of embodied interaction. To increase the perception of spatial presence, we extruded the thin map to the third dimension, creating a three-dimensional object, and added diffuse reflection shading to the extruded base of the map. The rendering of flows was modified to use curved tubes rather than the computationally simpler and faster twodimensional bands to give the flow lines a three-dimensional appearance.

The second proposed design consideration for embodied maps is consistent physics. Authors of immersive maps should apply a consistent set of physical laws, which are not required to replicate reality. In our immersive flow map, gravity does not exist, which allows the user to grab, move, and release a map to pin it in open space (Figure 3). Also, maps are not rigid but are instead scalable at will (Figure 8). To avoid interference with the colors on the choropleth map, a homogenous light source illuminates the scene, and objects do not cast shadows. Our map does not detect collisions among virtual objects. Hence, the user can defy real-world physics and arrange maps such that they intersect each other. This approach was chosen for convenience; in future work, rigid body physics could be simulated such that maps push each other away when they collide. The prototype also does not detect collisions between virtual maps and the physical environment; for example, when a map is thrown away, it sinks into the floor and disappears (Figure 6). 
The third design consideration is human body skills. It suggests that the physical skills of the user should be transferable to embodied maps. Body-based interaction with immersive maps has been suggested rarely in the literature. We propose a series of novel body-based interactions, for example, the shaking gesture for filtering data (Figure 9), or the throwing gesture for deleting a map (Figure 6). Inspired by the ImAxes immersive visualization framework, we enable users to link multiple maps with flow lines and also unlink flow maps (Figure 7). Controlling this proximity-based interaction requires users to move maps with their hands, and may also include walking a few steps. This is a direct transfer of the user's basic physical skills to an immersive visualization. However, we do not currently know how efficient or fatiguing these interactions are.

The fourth design consideration is direct manipulation. It suggests that an embodied map should support direct manipulation. We refrained from using user interface controls, such as buttons and sliders, and only designed direct manipulations for this exploration. We designed embodied interactions that use grabbing (Figure 3), throwing away (Figure 6), and shaking (Figure 9) for an entire map, and pushing away (Figure 10) as well as pulling out (Figure 5) individual flow line symbols. These interactions are engaging, but we do not currently know whether they are easy to detect and perform efficiently.

\section{Conclusion and Further Work}

We apply the concept of embodiment to immersive geovisualization and propose four design considerations for embodied geovisualization and embodied interactions: (1) Object Presence: The user of a map in virtual reality or augmented reality should perceive the map and its elements as objects that are present; (2) consistent physics: the map should follow a set of physical laws; (3) human body skills: the user's basic physical skills should be transferrable; and (4) direct manipulation: the map should support direct manipulation for map-specific operations. We hope that authors of immersive maps and other types of geovisualizations will find the four proposed design considerations helpful for creating embodied gesture interactions. As gesture tracking and immersive visualization technology mature, we expect these design considerations to evolve and grow in number.

We present an example of an interactive immersive flow map that provides a sense of presence, and a series of novel embodied interactions. Using this map as an illustrative example of an embodied immersive maps, we describe a set of hand gestures that are mapped to work and enabling operators.

Büschel et al. (2018) argued that the goal of a user interface is to minimize the cognitive distance between a user's intent and the execution of that intent by the system (which was originally defined as the gulf of execution by Norman (1988)). Our example flow map uses 
embodiment for direct manipulation of the data and the visualization. For example, users can create flows between two maps with direct embodied interaction by grabbing the maps and moving them sufficiently close to each other. This differs from conventional interaction in which two maps are selected before an indirect user interface element, such as a menu or a button, is activated to create the flow lines. It is reasonable to expect that the more direct embodied interaction reduces the gap between the user's intent and the execution of the action, however, the ease of learning and effectiveness remain to be evaluated through user studies.

We focused on a limited set of embodied interactions that directly manipulate the map. We did not develop interactions for other operators, such as annotate, resymbolize, overlay, or search operators (Roth, 2013), which traditionally use interface widgets like text fields for entering query terms or buttons to confirm selections. One possible approach for enabling direct manipulation and avoiding traditional interface elements could use interactive legend widgets. It remains to be explored whether "smart legends" as proposed by Sieber et al. (2005) and Cron et al. (2008) can be brought to immersive cartography and how they can enable additional direct embodied interaction operators.

While we explored the design space of embodied interactions for immersive maps by the example of flow maps, we believe that the proposed embodied interactions should be generalizable to other types of immersive maps and geovisualizations. For example, the shaking interaction can be generalized to filter other types of quantitative map symbols. The pulling of flow map symbols can also be generalized to other map symbols, for example, a volume of an embodied prism map could be pulled out to obtain further details about that region. Another example is the ability to push away symbols in a cluttered environment, which can also be applied to other map symbols, such as bar columns placed on an immersive map.

While the exploratory immersive map is manipulated with standard hand-held VR controllers, all presented embodied interactions should be transferable to freehand interactions using trackers that are, for example, integrated in the recent Oculus Quest and Microsoft HoloLens 2 headsets. It would also be interesting to explore how these embodied interactions can be applied to mobile maps considering that most of the mobile devices support basic gestures recognition (Büschel et al., 2019; Spindler et al., 2014).

We acknowledge that a limitation of our work is the lack of user studies to assess (1) the validity of the proposed design considerations, (2) the effectiveness of the proposed embodied gesture interactions, (3) the transferability of the proposed interactions to other types of maps and geovisualizations, and (4) effectiveness of immersive analytics with embodied maps and gesture interaction for knowledge discovery.

We used an immersive flow map to explore embodied interaction with maps in virtual reality. Our aim was not to evaluate the effectiveness of immersive flow maps, compare them to 
conventional flow maps, or evaluate the effectiveness of design variations of such maps. Nevertheless, our exploration led us to identify open questions and future research directions related to flow mapping in immersive space. For example, we were unsure how to best visualize flow direction. We found three-dimensional arrowheads to be bulky and their esthetics debatable. We instead used a color gradient to indicate direction because this technique is simple to implement - but perhaps particles moving along the flow lines would have been more effective to show direction (Romat et al., 2018)? Or would this be distractive? Another open question is whether our linked flow maps are useful. Yang et al. (2019) found that their related immersive MapsLink design was not successful. MapsLink duplicates a map to show direction: all flow lines start on one map and end on the other map, while both maps show the same geographic area. Our linked flow maps differ, as the geographic areas are not duplicated, and origins and destinations can be located on the same map. Whether our design is efficient and effective remains to be evaluated. Another interesting question relates to adjusting the display of flow lines to the current area of interest to reduce "visual clutter" (Schöttler et al., 2021). Visual clutter is a major issue for complex flow maps (and other network visualizations) and is often due to long flow lines crossing an area of interest: if the source and the destination of a flow line are invisible because they are located outside of a relatively small focus area, then the line does not convey any useful information and is nothing than distracting noise. With gaze tracking technology integrated into head-mounted displays, the displayed flow lines could be automatically adjusted such that lines starting and ending outside the central focus area are not rendered.

Finallywith the rapid advancement of hand tracking technology and head-mounted displays for VR and AR, an increasing number of people will create and use diverse types of embodied objects in the future. It is reasonable to expect that interaction standards with embodied general-purpose objects will be established. These interaction conventions will influence how embodied maps will be manipulated. However, there is little published research currently exploring embodied interaction with immersive maps, and there is considerable room for designing new embodied interaction paradigms for immersive maps and geovisualizations.

\section{Acknowledgements}

The authors would like to thank the anonymous reviewers for their valuable comments and suggestions, and Jane Darbyshire (Esri, Redlands) for editing this article.

\section{References}

Austin, C. R., Ens, B., Satriadi, K. A., \& Jenny, B. (2020). Elicitation study investigating hand 
and foot gesture interaction for immersive maps in augmented reality. Cartography and Geographic Information Science, 47(3), 214-228.

https://doi.org/10.1080/15230406.2019.1696232

Batch, A., Cunningham, A., Cordeil, M., Elmqvist, N., Dwyer, T., Thomas, B. H., \& Marriott, K. (2020). There is no spoon: evaluating performance, space use, and presence with expert domain users in immersive analytics. IEEE Transactions on Visualization and Computer Graphics, 26(1), 536-546. https://doi.org/10.1109/TVCG.2019.2934803

Bilke, L., Fischer, T., Helbig, C., Krawczyk, C., Nagel, T., Naumov, D., Paulick, S., Rink, K., Sachse, A., Schelenz, S., Walther, M., Watanabe, N., Zehner, B., Ziesch, J., \& Kolditz, O. (2014). TESSIN VISLab - laboratory for scientific visualization. Environmental Earth Sciences, 72(10), 3881-3899. https://doi.org/10.1007/s12665-014-3785-5

Bruder, G., Steinicke, F., \& Hinrichs, K. H. (2009). Arch-explore: A natural user interface for immersive architectural walkthroughs. Proceedings of IEEE Symposium on 3D User Interfaces 3DUI, 75-82. https://doi.org/10.1109/3DUI.2009.4811208

Büschel, W., Chen, J., Dachselt, R., Drucker, S., Dwyer, T., Görg, C., Isenberg, T., Kerren, A., North, C., \& Stuerzlinger, W. (2018). Interaction for immersive analytics. In K. Marriott, F. Schreiber, T. Dwyer, K. Klein, N. H. Riche, T. Itoh, W. Stuerzlinger, \& B. H. Thomas (Eds.), Immersive analytics (pp. 95-138). Springer. https://doi.org/10.1007/978-3-03001388-2_4

Büschel, W., Mitschick, A., Meyer, T., \& Dachselt, R. (2019). Investigating smartphone-based pan and zoom in 3D data spaces in augmented reality. Proceedings of the 21st International Conference on Human-Computer Interaction with Mobile Devices and Services, MobileHCI 2019. https://doi.org/10.1145/3338286.3340113

Butscher, S., Hubenschmid, S., Müller, J., Fuchs, J., \& Reiterer, H. (2018). Clusters, trends, and outliers: How Immersive technologies can facilitate the collaborative analysis of multidimensional data. Conference on Human Factors in Computing Systems Proceedings, 2018-April. https://doi.org/10.1145/3173574.3173664

Chandler, T., Cordeil, M., Czauderna, T., Dwyer, T., Glowacki, J., Goncu, C., Klapperstueck, M., Klein, K., Marriott, K., Schreiber, F., \& Wilson, E. (2015). Immersive analytics. Proceedings of Big Data Visual Analytics BDVA 2015, 1-8. https://doi.org/10.1109/BDVA.2015.7314296 
Çöltekin, A., Lochhead, I., Madden, M., Christophe, S., Devaux, A., Pettit, C., Lock, O., et al. (2020). Extended reality in spatial sciences: a review of research challenges and future directions. ISPRS International Journal of Geo-Information, 9(7), 439. https://doi.org/10.3390/ijgi9070439

Cordeil, M., Bach, B., Cunningham, A., Montoya, B., Smith, R. T., Thomas, B. H., \& Dwyer, T. (2020). Embodied Axes: tangible, actuated interaction for 3D augmented reality data spaces. Proceedings of the 2020 CHI Conference on Human Factors in Computing Systems, 1-12. https://doi.org/10.1145/3313831.3376613

Cordeil, M., Cunningham, A., Bach, B., Hurter, C., Thomas, B. H., Marriott, K., \& Dwyer, T. (2019). IATK: An immersive analytics toolkit. Proceedings of the 26th IEEE Conference on Virtual Reality and 3D User Interfaces, 200-209. https://doi.org/10.1109/VR.2019.8797978

Cordeil, M., Cunningham, A., Dwyer, T., Thomas, B. H., \& Marriott, K. (2017). ImAxes: Immersive axes as embodied affordances for interactive multivariate data visualisation. Proceedings of the 30th Annual ACM Symposium on User Interface Software and Technology UIST, 71-83. https://doi.org/10.1145/3126594.3126613

Cron J., Wiesmann S., Hurni L. (2008). Facilitating the handling of interactive atlases by dynamic grouping of functions - the example of "smart legend". In: Moore A., Drecki I. (eds) Geospatial Vision. Lecture Notes in Geoinformation and Cartography (pp. 1-18). Springer, Berlin, Heidelberg. https://doi.org/10.1007/978-3-540-70970-1_1

Davies, C. (1998). Analysing 'work' in complex system tasks: an exploratory study with GIS. Behaviour \& Information Technology, 17(4), 218-230. https://doi.org/10.1080/014492998119427

Dourish, P. (2001). Where the action is: the foundations of embodied interaction. MIT Press. https://doi.org/10.7551/mitpress/7221.001.0001

Dwyer, T., Marriott, K., Isenberg, T., Klein, K., Riche, N., Schreiber, F., Stuerzlinger, W., \& Thomas, B. H. (2018). Immersive analytics: an introduction. In K. Marriott, F. Schreiber, T. Dwyer, K. Klein, N. H. Riche, T. Itoh, W. Stuerzlinger, \& B. H. Thomas (Eds.), Immersive analytics: Vol. 11190 LNCS (pp. 1-23). Springer. https://doi.org/10.1007/9783-030-01388-2_1

Elmqvist, N., Moere, A. Vande, Jetter, H.-C., Cernea, D., Reiterer, H., \& Jankun-Kelly, T. 
(2011). Fluid interaction for information visualization. Information Visualization, 10(4), 327-340. https://doi.org/10.1177/1473871611413180

Ens, B., Bach, B., Cordeil, M., Engelke, U., Serrano, M., Prouzeau, A., Anthes, C., Büschel, W., Dunne, C., Dwyer, T., Grubert, J., Haga, J. H., Kirshenbaum, N., Kobayashi, D., \& Lin, T. (2021). Grand challenges in immersive analytics. Proceedings of the $2021 \mathrm{CHI}$ Conference on Human Factors in Computing Systems. https://doi.org/10.1145/3411764.3446866

Ens, B., Goodwin, S., Prouzeau, A., Anderson, F., Wang, F. Y., Gratzl, S., Lucarelli, Z., Moyle, B., Smiley, J., \& Dwyer, T. (2021). Uplift: a tangible and immersive tabletop system for casual collaborative visual analytics. IEEE Transactions on Visualization and Computer Graphics, 27(2), 1193-1203. https://doi.org/10.1109/tvcg.2020.3030334

Falconer, C. J., Slater, M., Rovira, A., King, J. A., Gilbert, P., Antley, A., \& Brewin, C. R. (2014). Embodying compassion: A virtual reality paradigm for overcoming excessive selfcriticism. PLoS ONE, 9(11). https://doi.org/10.1371/journal.pone.0111933

Fishkin, K. P., Moran, T. P., \& Harrison, B. L. (1998). Embodied user interfaces: towards invisible user interfaces. Proceedings of IFIP International Conference on Engineering for Human-Computer Interaction, 1-18. https://doi.org/10.1007/978-0-387-35349-4_1

Giannopoulos, I., Komninos, A., \& Garofalakis, J. (2017). Natural interaction with large map interfaces in VR. Proceedings of the 21st Pan-Hellenic Conference on Informatics, 1-6. https://doi.org/10.1145/3139367.3139424

Hedley, N. (2015). Virtual reality. In M. Monmonier (Ed.), The History of Cartography: Cartography in the Twentieth Century (Vol. 6, pp. 1680-1683). University of Chicago Press.

Hedley, N. (2017). Augmented reality. In International Encyclopedia of Geography: People, the Earth, Environment and Technology (pp. 1-13). John Wiley \& Sons, Ltd. https://doi.org/10.1002/9781118786352.wbieg0961

Hurter, C., Riche, N., Drucker, S., Cordeil, M., Vuillemot, R., Hurter, C., Riche, N., Drucker, S., Cordeil, M., Alligier, R., Hurter, C., Riche, N. H., Drucker, S. M., Cordeil, M., Alligier, R., \& Vuillemot, R. (2018). FiberClay: sculpting three dimensional trajectories to reveal structural insights. IEEE Transactions on Visualization and Computer Graphics, 25(1), 704-714. https://doi.org/10.1109/TVCG.2018.2865191 
Jacob, R. J., Girouard, A., Hirshfield, L. M., Horn, M. S., Shaer, O., Solovey, E. T., \& Zigelbaum, J. (2008). Reality-based interaction : a framework for post-WIMP interfaces. Proceedings of the SIGCHI Conference on Human Factors in Computing Systems, 201210. https://doi.org/10.1145/1357054.1357089

Jenny, B., Stephen, D. M., Muehlenhaus, I., Marston, B. E., Sharma, R., Zhang, E., \& Jenny, H. (2018). Design principles for origin-destination flow maps. Cartography and Geographic Information Science, 45(1), 62-75. https://doi.org/10.1080/15230406.2016.1262280

Jerald, J. (2015). The VR book: Human-centered design for virtual reality. Morgan \& Claypool. https://doi.org/10.1145/2792790

Kilteni, K., Groten, R., \& Slater, M. (2012). The sense of embodiment in virtual reality. Presence: Teleoperators and Virtual Environments, 21(4), 373-387. https://doi.org/10.1162/PRES_a_00124

Klippel, A. (2020). From spatial to platial - the role and future of immersive technologies in the spatial sciences. Journal of Spatial Information Science, 21(21), 33-45. https://doi.org/10.5311/josis.2020.21.722

Laha, B., Sensharma, K., Schiffbauer, J. D., \& Bowman, D. A. (2012). Effects of immersion on visual analysis of volume data. IEEE Transactions on Visualization and Computer Graphics, 18(4), 597-606. https://doi.org/10.1109/TVCG.2012.42

Lee, B., Hu, X., Cordeil, M., Prouzeau, A., Jenny, B., \& Dwyer, T. (2021). Shared surfaces and spaces: collaborative data visualisation in a co-located immersive environment. IEEE Transactions on Visualization and Computer Graphics, 27(2), 1171-1181. https://doi.org/10.1109/TVCG.2020.3030450

Lee, K. M. (2004). Presence, explicated. Communication Theory, 14(1), 27-50. https://doi.org/10.1111/j.1468-2885.2004.tb00302.x

Marriott, K., Chen, J., Hlawatsch, M., Itoh, T., Nacenta, M. A., Reina, G., \& Stuerzlinger, W. (2018). Immersive analytics: time to reconsider the value of $3 \mathrm{~d}$ for information visualisation. In K. Marriott, F. Schreiber, T. Dwyer, K. Klein, N. H. Riche, T. Itoh, W. Stuerzlinger, \& B. H. Thomas (Eds.), Immersive analytics: Vol. 11190 LNCS (pp. 25-55). Springer Verlag. https://doi.org/10.1007/978-3-030-01388-2_2

Mine, M. R., Brooks, Jr., F. P., \& Sequin, C. H. (1997). Moving objects in space: exploiting proprioception In virtual-environment interaction. Proceedings of ACM SIGGRAPH '97, 
Nam, J. W., McCullough, K., Tveite, J., Espinosa, M. M., Perry, C. H., Wilson, B. T., \& Keefe, D. F. (2019). Worlds-in-wedges: Combining worlds-in-miniature and portals to support comparative immersive visualization of forestry data. Proceedings of the 26th IEEE Conference on Virtual Reality and 3D User Interfaces, VR 2019, 747-755. https://doi.org/10.1109/VR.2019.8797871

Norman, D. A. (1988). The psychology of everyday things. In The psychology of everyday things. Basic Books.

Norman, D. A. (2013). The design of everyday things. Basic Books.

Prouzeau, A., Lhuillier, A., Ens, B., Weiskopf, D., \& Dwyer, T. (2019). Visual link routing in immersive visualisation. ISS 2019 - Proceedings of the 2019 ACM International Conference on Interactive Surfaces and Spaces, 241-253. https://doi.org/10.1145/3343055.3359709

Prouzeau, A., Wang, Y., Ens, B., Willett, W., \& Dwyer, T. (2020). Corsican twin: authoring in situ augmented reality visualisations in virtual reality. Proceedings of the International Conference on Advanced Visual Interfaces, 1-9. https://doi.org/10.1145/3399715.3399743

Quach, Q., \& Jenny, B. (2020). Immersive visualization with bar graphics. Cartography and Geographic Information Science, 47(6), 471-480. https://doi.org/10.1080/15230406.2020.1771771

Romat, H., Appert C., Bach, B., Henry-Riche, N., \& Pietriga, E. (2018). Animated edge textures in node-link diagrams: a design space and initial evaluation. Proceedings of the $2018 \mathrm{CHI}$ Conference on Human Factors in Computing Systems (CHI '18). Association for Computing Machinery, New York, NY, USA, Paper 187, 1-13. https://doi.org/10.1145/3173574.3173761

Roth, R. E. (2013). An empirically-derived taxonomy of interaction primitives for interactive cartography and geovisualization. IEEE Transactions on Visualization and Computer Graphics, 19(12), 2356-2365. https://doi.org/10.1109/TVCG.2013.130

Santos-Torres, A., Zarraonandia, T., Díaz, P., \& Aedo, I. (2018). Exploring interaction mechanisms for map interfaces in virtual reality environments. Proceedings of the XIX International Conference on Human Computer Interaction (Interacción 2018), 1-7. https://doi.org/10.1145/3233824.3233828 
Satriadi, K. A., Ens, B., Cordeil, M., Czauderna, T., \& Jenny, B. (2020). Maps around me: 3D multiview layouts in immersive spaces. Proceedings of the ACM Conference on Interactive Surfaces and Spaces ISS, 4, 20. https://doi.org/10.1145/3427329

Satriadi, K. A., Ens, B., Cordeil, M., Jenny, B., Czauderna, T., \& Willett, W. (2019). Augmented reality map navigation with freehand gestures. Proceedings of the 26th IEEE Conference on Virtual Reality and 3D User Interfaces, VR 2019, 593-603. https://doi.org/10.1109/VR.2019.8798340

Schöttler, S., Yang, Y., Pfister, H., \& Bach, B. (2021). Visualizing and interacting with geospatial networks: A survey and design space. Computer Graphics Forum. https://doi.org/10.1111/cgf.14198

Sieber, R., Schmid, C., and Wiesmann, S. (2005). Smart legend-smart atlas. Proceedings of the 22th International Cartographic Conference ICC.

Shneiderman, B., Plaisant, C., Cohen, M., Jacobs, S., Elmqvist, N., \& Diakopoulos, N. (2016). Designing the user interface: strategies for effective human-computer interaction. Pearson.

Sicat, R., Li, J., Choi, J., Cordeil, M., Jeong, W. K., Bach, B., \& Pfister, H. (2019). DXR: A toolkit for building immersive data visualizations. IEEE Transactions on Visualization and Computer Graphics, 25(1), 715-725. https://doi.org/10.1109/TVCG.2018.2865152

Slater, M., \& Wilbur, S. (1997). A framework for immersive virtual environments (FIVE): Speculations on the role of presence in virtual environments. Presence: Teleoperators and Virtual Environments, 6(6), 603-616. https://doi.org/10.1162/pres.1997.6.6.603

Spindler, M., Schuessler, M., Martsch, M., \& Dachselt, R. (2014). Pinch-drag-flick vs. spatial input: rethinking zoom and pan on mobile displays. Proceedings of the 32nd Annual ACM Conference on Human Factors in Computing Systems, 1113-1122. https://doi.org/10.1145/2556288.2557028

Spur, M., Tourre, V., David, E., Moreau, G., \& Le Callet, P. (2020). MapStack: Exploring multilayered geospatial data in virtual reality. Proceedings of the 11th International Conference on Information Visualization Theory and Applications, 88-99. https://doi.org/10.5220/0008978600880099

Stephen, D. M., \& Jenny, B. (2017). Automated layout of origin-destination flow maps: U.S. county-to-county migration 2009-2013. Journal of Maps, 13(1), 46-55. 
https://doi.org/10.1080/17445647.2017.1313788

U.S. Census Bureau (2020). County-to-county migration flows.

https://www.census.gov/topics/population/migration/guidance/county-to-countymigration-flows.html

Wagner Filho, J. A., Stuerzlinger, W., \& Nedel, L. (2020). Evaluating an immersive space-time cube geovisualization for intuitive trajectory data exploration. IEEE Transactions on Visualization and Computer Graphics, 26(1), 514-524.

https://doi.org/10.1109/TVCG.2019.2934415

Walsh, J., Cunningham, A., Smith, R., \& Thomas, B. (2018). Tangible braille plot: Tangibly exploring geo-temporal data in virtual reality. Proceedings of the International Symposium on Big Data Visual and Immersive Analytics BDVA. https://doi.org/10.1109/BDVA.2018.8534024

Ware, C. (2012). Information visualization: perception for design. Morgan Kaufmann.

Wigdor, D., \& Wixon, D. (2011). Brave NUI world: designing natural user interfaces for touch and gesture. Elsevier.

Whitefield, A., Escgate, A., Denley, I., \& Byerley, P. (1993). On distinguishing work tasks and enabling tasks. Interacting with Computers, 5(3), 333-347. https://doi.org/10.1016/09535438(93)90014-K

Witmer, B. G., \& Singer, M. J. (1998). Measuring presence in virtual environments: A presence questionnaire. Presence: Teleoperators and Virtual Environments, 7(3), 127-132. https://doi.org/10.1162/105474698565686

Yang, Y., Dwyer, T., Jenny, B., Marriott, K., Cordeil, M., \& Chen, H. (2019). Origindestination flow maps in immersive environments. IEEE Transactions on Visualization and Computer Graphics, 25(1), 693-703. https://doi.org/10.1109/TVCG.2018.2865192

Yang, Y., Dwyer, T., Marriott, K., Jenny, B., \& Goodwin, S. (2020). Tilt Map: interactive transitions between 2D choropleth map, 3D prism map and bar chart in immersive environments. IEEE Transactions on Visualization and Computer Graphics. https://doi.org/10.1109/TVCG.2020.3004137

Yang, Y., Jenny, B., Dwyer, T., Marriott, K., Chen, H., \& Cordeil, M. (2018). Maps and globes in virtual reality. Computer Graphics Forum, 37(3), 427-438. 
https://doi.org/10.1111/cgf.13431

Zhang, M.-J., Li, J., \& Zhang, K. (2016). An immersive approach to the visual exploration of geospatial network datasets. Proceedings of the 15th ACM SIGGRAPH Conference on Virtual-Reality Continuum and Its Applications in Industry - VRCAI '16, 381-390. https://doi.org/10.1145/3013971.3013983

Zhang, M.-J., Zhang, K., Li, J., \& Li, Y. N. (2018). Visual exploration of 3D geospatial networks in a virtual reality environment. Computer Journal, 61(3), 447-458. https://doi.org/10.1093/comjnl/bxx117 